\title{
The Relation between Calcium Supplement Consumption and Calcific Shoulder Tendonitis
}

\author{
Alireza Rouhani ${ }^{1}$, Jafar Soleimanpour ${ }^{1}$, Ayaz Sadeghilar $^{1^{*}}$, Ali Tabrizi $^{1}$
}

1. Department of Orthopedic Surgery, Tabriz University of Medical Sciences, Tabriz, Iran

\begin{abstract}
Background: Calcific tendonitis is a common cause of non-traumatic shoulder pain. Previous studies have suggested a relation between minerals and endocrine and calcium deposition. Thus, hypercalcemia is probably related to calcific tendonitis. This study aims at evaluating the relation found between calcium supplement consumption and calcific shoulder tendonitis. Methods: This analyticaldescriptive study was conducted on 250 patients with shoulder pain referring to clinics and emergency department of Shohada Orthopedics Hospital during one year for considering calcific shoulder tendonitis and calcium supplement consumption. Patients with calcific tendonitis were treated and their functional ability was evaluated using DASH questionnaire, pain severity and range of motion (ROM) before and after treatment and their correlation with calcium supplement consumption. Results: Calcific tendonitis and calcium consumption were generally seen in $30(12 \%)$ and $73(29.2 \%)$ cases, respectively. Calcium consumption frequency in patients with calcific tendonitis was significantly higher than the patients who did not consume calcium supplements ( $76.7 \%$ vs. $22.7 \%)$. Patients with calcific tendonitis who did not consume calcium supplements suffered from significantly longer periods of shoulder pain. All patients having consumed calcium supplement were female. The group who consumed calcium supplement had significantly severe pain and higher DASH score before and after treatment, while there was no significant difference in number of impaired ROM before and after treatment. Also, there was a negative correlation between calcium supplement consumption, pain severity and DASH score before and after treatment. Conclusion: Calcium supplement consumption is related to calcific tendonitis and is also accompanied with more pain and lower functional ability in patients with calcific tendonitis.
\end{abstract}

Keywords: Calcific tendonitis; Shoulder; Calcium supplement; Pain

\section{Introduction}

Calcific tendonitis is a common cause of nontraumatic shoulder pain $(1,2)$. Regional deposition of calcium at rotator cuff tendon results in shoulder calcific tendonitis,

inflammation and some complications such as painful shoulder movements $(1,2)$. The disease is detected through clinical examination and imaging techniques. Biochemical and hematological tests play no role in diagnosis

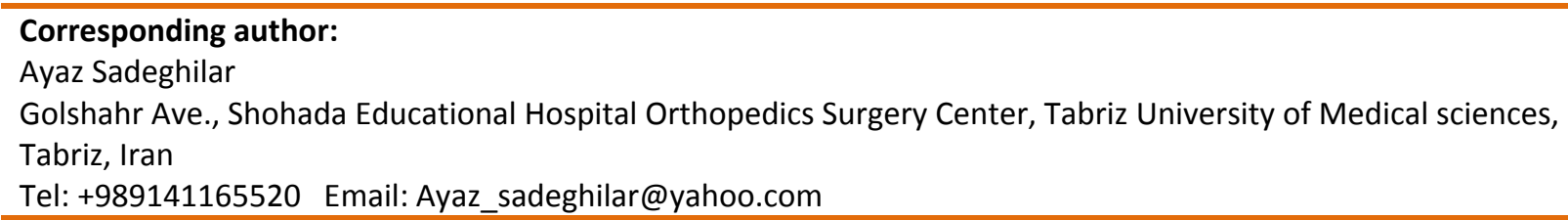


process of the disease $(2,3)$. Calcium deposits cyclically and is divided into three main stages: 1) pre-calcific which lacks any clinical symptoms and pain; 2) calcific which is divided into three formative, resting, and resorptive phases; and 3) post-calcific which includes repair phase and the patient may suffer from pain (4).

Etiology and pathogenesis of shoulder calcific tendonitis is not already known and several studies were conducted on different factors (1-3). It is observed that the more the calcium deposition in shoulder, the more the disease severity (5). Thus, it is possible to prevent from disease or reduce its severity through controlling possible factors which increase calcium level. Previous studies suggested a relation between status of body minerals and endocrine and calcium calcification. Higher prevalence of thyroid and steroid hormone variations in patients suffering from calcific tendinitis has been reported in comparison with healthy subjects (6). Additionally, it has been stated that a transient hyperparathyroidism and a subsequent secondary hypercalcemia to vitamin D insufficiency may result in calcification in shoulder tendonitis (7). Therefore, it is possible that nutritional supplements increasingly consumed by patients may be involved in initiation/intensification of symptoms or progression of the disease. The present study was designed to evaluate the relation found between calcium supplements consumption and shoulder calcific tendinitis.

\section{Materials and methods}

In an analytical-descriptive study, 250 patients with shoulder calcific tendinitis were identified. All patients were studied considering basic findings as well as consumption of calcium supplements and other possible etiologies and frequency of tendinitis and supplements consumption was determined. Initially, clinical examinations, CT scan, and radiological findings were evaluated and recorded in the checklist. Range of motion of shoulder and pain severity of the patients was studied. History of calcium supplements consumption was evaluated and recorded considering dosage and duration. Additionally, functional ability of the patients suffering from calcific tendinitis was studied before and after surgery using DASH score.

Therapeutic results of calcium supplements in consumers and subjects of the control group (without consumption of these supplements) were compared within a three-month follow-up period. Pain severity during follow-up period was evaluated using Visual Analog Scale when the patients referred to the center. DASH is a 30-item questionnaire designed to measure physical symptoms and function of the patients suffering from musculoskeletal disorders. The questionnaire evaluates functional status of upper limb of the patient within the last one week. The questionnaire includes items to measure problems of the patient in routine affairs (21 questions), pain severity during sleep and activity and joint stiffness (5 questions), and role of upper limbs in social and occupational activities (4 questions). To obtain useful results, the patient should answer at least 27 questions out of 30 ones. Score of the questionnaire is 100 and the more the score close to 100 , the more disabled the patient. Validity and reliability of the questionnaire has been approved in Iranian medical societies (8).

Range of motion of the shoulder was measured in main motions such as flexion, extension, internal rotation, and external rotation using goniometer and the results were classified as normal or reduced ROM before and after treatment.

\section{Statistical analysis}

All understudy data were analyzed using SPSS.17 statistical software and descriptive statistical methods such as frequency and percentage. To compare quantitative findings 
between two groups, Independent T-test and Mann-Whitney $U$ test were used for normal and abnormal distributions, respectively. Also, Chi square test and Fischer exact test (if required) were used to evaluate qualitative findings. In this study, $\mathrm{P}<0.05$ was considered as statistically significant.

\section{Results}

The present study was conducted on 250 patients (73 male $(29.2 \%)$ and $177(70.8 \%)$ male and female, respectively) suffering from shoulder pain. The patients were qualified to enter the study which was conducted for one year. Age average of the patients was $54.50 \pm 9.06$ years (median=54). The youngest and oldest patients were 26 and 78 years old, respectively. Calcific tendinitis was seen in 30 (12\%) patients. Additionally, calcium consumption was reported in 73 (29.2\%) patients. Age average of the patients in the calcific tendinitis group and the control group was $52.30 \pm 11.94$ and $54.80 \pm 8.58$ years, respectively. There was not any statistically significant difference between these groups $(P=0.15)$.

Frequency of calcium supplements consumption was significantly higher in patients suffering from calcific tendinitis compared to without tendinitis, $76.7 \%$ versus $22.7 \%(p<0.001)$. Average dosage of calcium consumption was determined in subjects of both groups with history of calcium consumption. It was $14.43 \pm 13.24$ and $13.34 \pm 9.99$ months in the groups with and without tendinitis, respectively. Although calcium was consumed for shorter periods in tendinitis group, the difference was not statistically significant $(p=0.27)$.

Table 1 shows basic findings in the patients suffering from calcific tendinitis with and without consumption of calcium supplements. As seen, shoulder pain arising from calcific tendinitis lasted for longer periods in the group which did not consume calcium supplement. All cases of calcium supplements consumption were female.

The patients suffering from tendinitis underwent physiotherapy accompanied by NSAID. All patients responded to the treatment process and surgery was not required to mitigate the pain or improve shoulder function. VAS scale was used to measure pain severity

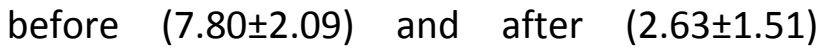
treatment. The difference was statistically significant $(P<0.001)$. Functional point of DASH was $60.43 \pm 10.12$ before and $23.00 \pm 10.77$ after treatment which was significantly improved after treatment $(P<0.001)$. There was reduced ROM in 20 (66.7\%) subjects before treatment and it was reduced to 4 subjects (13.3\%) after treatment.

Table 2 refers to pain severity, physical function status, and range of motion before and after treatment in the groups with and without calcium supplement consumption. According to this table, patients consuming calcium supplements experienced more severe pain and higher DASH score before and after treatment in comparison with the group without calcium supplement consumption. In spite of more restricted range of motion before and after treatment in the group with calcium supplement consumption, the difference was not statistically significant. The relation between calcium consumption and pain severity, DASH score, and ROM before and after surgery was determined using Pearson coefficient. Statistically, there was a significant relation between calcium consumption and pain severity before ( $r=-0.40$ and $P=0.02)$ and after $(r=-0.44, P=0.01)$ treatment, and DASH score before $(r=-0.365, P=0.04)$ and after $(r=-$ 0.379, $\mathrm{P}=0.03$ ) treatment. ROM was studied after treatment as response rate to treatment and there was not observed any significant statistical relation $(r-=0.216, P=0.25)$. 


\begin{tabular}{llccl}
\hline & & $\begin{array}{c}\text { With supplement } \\
\text { consumption }\end{array}$ & $\begin{array}{c}\text { Without supplement } \\
\text { consumption }\end{array}$ & P value \\
\hline Age & $53.69 \pm 11.46$ & $47.71 \pm 13.25$ & 0.25 \\
Gender & Male & 0 & $3(42.9 \%)$ & 0.009 \\
Duration of shoulder pain & Female & $23(100 \%)$ & $4(57.1 \%)$ & \\
\hline
\end{tabular}

Table 1: Basic findings in patients suffering from calcific tendinitis with and without calcium supplements consumption

\begin{tabular}{llccc}
\hline & With supplement & $\begin{array}{c}\text { Without supplement } \\
\text { consumption }\end{array}$ & P value \\
\hline Pain severity (VAS) & Before & $8.26 \pm 2.07$ & $6.28 \pm 1.38$ & 0.02 \\
& After & $3.00 \pm 1.47$ & $1.42 \pm 0.97$ & 0.01 \\
DASH score & Before & $62.43 \pm 10.18$ & $53.85 \pm 10.98$ & 0.04 \\
& After & $25.21 \pm 10.48$ & $15.71 \pm 8.80$ & 0.03 \\
Reduced ROM & Before & $15(65.2 \%)$ & $4(57.1 \%)$ & 0.69 \\
& After & $4(17.4 \%)$ & 0 & 0.54 \\
\hline
\end{tabular}

Table 2: Pain severity, physical function status, and range of motion before and after treatment between subjects with and without calcium supplement consumption

\section{Discussion}

Calcific tendonitis is a common cause of nontraumatic shoulder pain (9-12) and is prevalently seen in old patients $(13,14)$. Several studies have reported different frequencies of calcific tendinitis. Its prevalence in non-symptomatic subjects was $2.7-7.5 \%$ and in the symptomatic ones $6.5 \%(15,16)$.

In the present study, calcific tendinitis was observed in $12 \%$ of the patients referring with symptomatic shoulder pain. However, difference between volume of the understudy samples and time interval of the patients' evaluation might have affected the results. Calcium deposition at rotator cuff tendon results in shoulder calcific tendonitis, inflammation and painful symptoms $(8,9)$. As mentioned, etiologic factors of calcific tendinitis are still unknown and probably attributed to hypercalcemia in laboratory and clinical evaluations (17-19).

Halimi et al. suggested hypercalcemia as a probable factor in calcific tendinitis (19). According to Olszak et al., calcium deposition may lead to complications as well as chronic inflammatory variations in the damaged area, infection, and arteriosclerosis which have a significant relation with extracellular calcium as well as its consumption (17).

Chen et al. suggest that less calcium and calcium canals blocks may prevent from calcium deposition and calcific tendinitis through reducing extracellular calcium and preventing from its transfer into the cells (18). As seen in the present study, patients suffering from calcific tendinitis had significantly higher frequency considering calcium supplements consumption in comparison with subjects without tendinitis. Calcium supplement consumption may increase extracellular calcium level, calcium deposition risk, and calcific tendinitis. Similarly, Agrawal and Owen referred to probable role of post-graft hypercalcemia in appearing of calcific tendinitis in their study conducted on renal-grafted patients (20).

Confirming probable role of calcium and calcium supplements consumption, Yokoyama et al. demonstrated that Cimetidine decreases 
calcium deposition in tendons and improvement of patients suffering from calcific tendinitis through decreasing calcium level. Therefore, decrease of serum calcium level may prevent from reappearing and re-deposition of calcium (21). Although it is not easy to determine whether the pain is related to tendonitis or calcium depositions, acute pain appears when depositions are affected by absorption with phagocytosis $(8,17)$. It was observed that surgical methods well affect the patients suffering from calcific tendinitis. In this study, the patients underwent physiotherapy accompanied by NSAIDs. All patients responded to the treatment process and surgery was not required to mitigate the pain or improve shoulder function. Pain severity and physical function ability were significantly improved after treatment. Moreover, reduced ROM decreased from $66.7 \%$ to $13.3 \%$ after treatment. Similarly, Yokoyama et al. observed significant improvement considering pain severity and physical function disorder after nonsurgical treatment (21).

Evaluating effect of calcium supplement consumption, this study found a significantly negative relation between calcium consumption and pain severity and DASH score before and after treatment such that calcium consumers experienced more severe pain and inappropriate functional point. Although both groups responded to the treatment, the difference was still observed. According to Ogan et al., higher volume of calcium deposition was associated with inappropriate progress of the disease (22). Similarly, Chiou et al. observed a significant difference between the progressed cases considering the amount containing carbonate apatite (5).

\section{Conclusion}

Calcium supplement consumption is related to calcific tendonitis and is also accompanied with more pain and lower functional ability in patients with calcific tendonitis. Although exact mechanism of the relation is yet unknown, restricting calcium consumption especially in patients with history of shoulder pain may prevent from calcific tendonitis and its undesired consequences.

\section{Acknowledgement}

This study was supported by Tabriz
University of

\section{References}

1. Kachewar SG, Kulkarni DS. Calcific tendinitis of the rotator cuff: a review. J Clin Diagn Res, 2013; 7(7):1482-5.

2. Suzuki K, Potts A, Anakwenze O, Singh A. Calcific Tendinitis of the Rotator Cuff: Management Options. J Am Acad Orthop Surg, 2014; 22(11):707-717.

3. Siegal DS, Wu JS, Newman JS, Del Cura JL, Hochman MG. Calcific tendinitis: a pictorial review. Can Assoc Radiol J, 2009; 60(5):263-72.

4. Uhthoff HK, Loehr JW. Calcific tendinopathy of the rotator cuff: pathogenesis, diagnosis, and management. J Am Acad Orthop Surg, 1997; 5:183-91.

5. Chiou HJ, Hung SC, Lin SY, Wei YS, Li MJ. Correlations among mineral components progressive calcification process and clinical symptoms of calcific tendonitis. Rheumatology, 2010; 49:548-555.

6. Harvie P, Pollard TC, Carr AJ. Calcific tendinitis: Natural history and association with endocrine disorders. J Shoulder Elbow Surg, 2007; 16:169-173.

7. Cavalli L, D’Elia G, Caracchini G, De Masi De Luca A, Innocenti P, Brandi ML. P28 - Calcific Tendinopathy and Vitamin D Status: A Potential Aetiopathogenetic Factor and Therapeutic Approach. Clinical Cases in Mineral and Bone Metabolism, 2010; 7(3):234. 
8. Mousavi SJ, Parnianpour M, Abedi M, Askary-Ashtiani A, Karimi A, Khorsandi A, et al. Cultural adaptation and validation of the Persian version of the Disabilities of the Arm, Shoulder and Hand (DASH) outcome measure. Clin Rehabil, 2008; 22(8):749-757.

9. Garcia GM, McCord GC, Kumar R. Hydroxyapatite crystal deposition disease. Semin Musculoskelet Radiol, 2003; 7:187-93.

10. Rogers LF, Hendrix RW. The painful shoulder. Radiol Clin North Am, 1988; 26(6):1359-71.

11. Hearnden A, Desai A, Karmegam A, Flannery M. Extracorporeal shock wave therapy in chronic calcific tendonitis of the shoulder--is it effective? Acta Orthop Belg, 2009; 75(1):25-31.

12. Burbank KM, Stevenson JH, Czarnecki GR, Dorfman J. Chronic shoulder pain: part I. Evaluation and diagnosis. Am Fam Physician, 2008; 77(4):453-60.

13. Hernandez-Santana A, Yavorsky A, Loughran ST, McCarthy GM, McMahon GP. New approaches in the detection of calcium-containing microcrystals in synovial fluid. Bioanalysis, 2011; 3(10):1085-91.

14. Tendinopathy. Greis AC, Derrington SM, McAuliffe M. Evaluation and Nonsurgical Management of Rotator Cuff Calcific. Orthop Clin North Am, 2015; 46(2):293-302.

15. Bureau NJ. Calcific tendinopathy of the shoulder. Semin Musculoskelet Radiol, 2013; 17(1):80-4.

16. Uhthoff HK, Sarkar K. Calcifying tendonitis. Baillieres Clin Rheumatol, 1989; 3:567-81.

17. Olszak IT, Poznansky MC, Evans RH, Olson D, Kos C, Pollak MR, et al. Extracellular calcium elicits a chemokinetic response from monocytes in vitro and in vivo. J Clin Invest, 2000; 105(9): 1299-305.

18. Chen W, Deng Y, Zhang J, Tang K. Uniaxial repetitive mechanical overloading induces influx of extracellular calcium and cytoskeleton disruption in human tenocytes. Cell Tissue Res, 2015; 359(2):577-87.

19. Halimi D, Benhamou CL, Amor B, Bricaire H, Luton JP. [Osteoarticular pathology, hypercalcemia and adrenal insufficiency. Analysis of 113 cases of adrenal insufficiency]. [Article in French] Ann Endocrinol (Paris), 1986; 47(6):4038.

20. Agarwal S, Owen R. Tendinitis and tendon ruptures in successful renal transplant recipients. Clin Orthop Relat Res, $1990 ; 252: 270-5$

21. Yokoyama M, Aono H, Takeda A, Morita K. Cimetidine for chronic calcifying tendinitis of the shoulder. Reg Anesth Pain Med, 2003; 28(3):248-52.

22. Ogon P, Suedkamp NP, M Jaeger, Izadpanah K, Koestler W, Maier D. Prognostic Factors in Nonoperative Therapy for Chronic Symptomatic Calcific Tendinitis of the Shoulder. Arthritis Rheum, 2009; 60(10):2978-84. 